\title{
COMPARACIÓN ENTRE LAS TÉCNICAS DE CITOLOGÍA COMPARTIDA: CONVENCIONAL VS. BASE LÍQUIDA
}

\author{
Piedad Campo Rodríguez MD*, Myriam Puerto de Amaya Bact, Cith**
}

\begin{abstract}
Resumen
Objetivo: describir las técnicas de citología cervicovaginal por el método convencional $v s$. base líquida, teniendo en cuenta la calidad de la muestra y los hallazgos morfológicos, en mujeres en edad reproductiva con vida sexual activa, tomadas en campañas de proyección social. Métodos: estudio descriptivo de corte transversal en 29 mujeres a quienes se tomaron 29 citologías, la mitad convencionales (extendidos sobre porta-objeto) y la otra mitad en base líquida. Se utilizó el método de muestra compartida (guardando la espátula y el citocepillo en el líquido fijador LucBase). Se excluyeron las embarazadas, las que estuvieron menstruando o que hubieran recibido radio o quimioterapia. Resultados: La variable sobre calidad de la muestra mostró que de las 29 convencionales 16 tenían material endocervical o de la zona de transformación, mientras en base líquida solo una tuvo endocérvix. En cuanto al registro de lectura, de 29 convencionales, tres fueron ASCUS; de catorce en base líquida, doce fueron inadecuadas y dos rechazadas. En lo referente al tiempo de realización de las técnicas, se halló una diferencia de siete minutos para la base líquida en relación con la convencional. Conclusiones: al comparar la muestra compartida para las dos técnicas, se encontró mayor celularidad y presencia de endocérvix en la convencional. Las preparaciones en base líquida mostraron fondo sucio constituido por células inflamatorias, moco y detritus celulares, no cumpliendo con una de las características de mejoramiento de la técnica. La muestra compartida haciendo primero el frotis convencional, reduce o acaba con el material que se preserva para la base líquida. Los nuevos estudios los enfocamos hacia el uso de nuevas tecnologías para base líquida como ThinPrep y SurePath.
\end{abstract}

Palabras clave: citología, base líquida, LucBase, ASCUS.

Abreviaturas: ASCUS, atipia de células escamosas de significado indeterminado.

\section{SHARED CONVENTIONAL VS LIQUID- BASED CYTOLOGY TECHNIQUES}

\begin{abstract}
Objective: to describe cervix cytology techniques, that is, the conventional method vs liquid- based preparations, taken during social projection campaigns, in terms of sample quality and morphologic findings, in sexually active women of reproductive age. Methods: a cross-sectional descriptive study in 29 women in which cytology was performed, half of them by the conventional method (smear on a slide) and the other half by a liquid-based preparation. The shared sample method was used (keeping the spatula and cytology brush in LucBase solution). Women who were pregnant, menstruating or had received chemotherapy or radiotherapy were excluded. Results: The variable on quality of the sample showed that of the 29 conventional samples, 16 had endocervical or transformation zone material, while only one on liquid-based preparation had endocervix. As to the reading records, out of 29 conventional samples, 3 were interpreted as ASCUS; out of 14 liquid-based, 12 were inadequate and two were rejected. A 7 minute difference in performing the liquid-based technique was found compared to the conventional technique. Conclusions: an increased cellularity and endocervix presence was found in the conventional technique when comparing the shared sample for the two techniques. Liquid-based preparations showed a dirty background consisting of inflammation, mucus and cellular detritus, not complying with one of the features of technique improvement. When performing the conventional smear first, the material preserved for the liquid-based technique is reduced or fade in the shared sample. Our new studies are focused on the use of new technologies for liquid- based preparations such as ThinPrep and SurePath.
\end{abstract}

Key words: cytology, liquid-based, LucBase, ASCUS. Abbreviations: ASCUS, atypical squamous cells of undetermined significance.

\footnotetext{
Fecha recibido: julio 6 de 2011 - Fecha aceptado: agosto 8 de 2011

* Médica Patóloga, Decana Facultad de Citohistología. Instructor Asociado, Fundación Universitaria de Ciencias de la Salud. Bogotá DC, Colombia.
}

** Instructor Asociado, Fundación Universitaria de Ciencias de la Salud. Bogotá DC. Colombia 


\section{Introducción}

En 1916 George Papanicolaou y Stockard analizaron los aspectos citomorfológicos del ciclo vaginal. En 1923 estudiaron el frotis vaginal en la mujer reconociendo por primera vez las células anormales del cáncer cervical. En 1943 publicaron una monografía sobre el cáncer cervicouterino en citología vaginal. ${ }^{1}$ Papanicolaou ideó un sistema de clasificación para las células exfoliadas en cinco grupos. 1) células dentro de límites normales, 2) atipias reactivas no sugestivas de malignidad, 3) células atípicas sugestivas pero no diagnósticas de malignidad, 4) células atípicas muy sospechosas de malignidad, 5) células conclusivas de malignidad. En 1973 la OMS (Organización Mundial de la Salud) estableció pautas para el diagnóstico citológico usando los conceptos de displasia y carcinoma in situ. En 1988 el Instituto Nacional de Salud de los Estados Unidos adoptó una terminología y una clasificación para reportar la citología cervical que fue llamada Sistema Bethesda, la cual no subclasificó a ASCUS, proponiendo la terminología de lesiones intraepiteliales cervicales de bajo y alto grado (LIEB y LIEA). ${ }^{\prime}$

En 1991 el término ASCUS se consideró como cambio reactivo en vez de LIEB, enfatizando que no cumple los parámetros para LEIB, sino que es una categoría intermedia. ${ }^{2}$ En 2001 el ASCUS se reclasificó como lesión escamosa intraepitelial de alto grado $(\mathrm{AG})$ o de bajo grado $(\mathrm{BG})$, rotulándose de la siguiente manera: LIESI (atipia de células escamosas de significado indeterminado sin poderse excluir lesión de alto grado) y LIEA (lesión intraepitelial escamosa de alto grado). ${ }^{2}$

En las técnicas de recolección de citología es posible obtener la muestra por dos métodos, la dividida y la de uso deseado. En la primera se obtiene el extendido convencional y el remanente se utiliza para citología en base líquida. En la segunda la muestra se coloca de inmediato en el medio fijador; en este tipo se pueden resolver los problemas del método convencional como son la captura y calidad de la totalidad de la muestra, fijación deficiente, distribución aleatoria de células anormales y existencia de elementos perturbadores. ${ }^{2}$
En el tamizaje del cáncer cervical la citología en base líquida es ahora muy utilizada a nivel mundial, remplazando la convencional en países industrializados, como sucede en el Reino Unido donde se emplea en el $85 \%$ de los laboratorios. Dentro de las ventajas de la base líquida encontramos la reducción de los resultados insatisfactorios y la alta tasa de detección de neoplasias intraepiteliales cervicales y glandulares. Sin embargo, algunos estudios reportan conflicto sugiriendo sensibilidad y especificidad equivalente para la detección de neoplasias intraepiteliales de alto grado. ${ }^{3}$ Según estudios realizados la sensibilidad del extendido convencional es del $82 \%$, base líquida de $86 \%$, especificidad $40 \%$ y $43 \%$; existe gran sensibilidad para el diagnóstico de neoplasias cervicales en base líquida con el ThinPrep que detecta lesiones intraepiteliales de alto grado LIEA. ${ }^{4}$

La citología convencional presenta limitaciones dadas por factores como extendido hemático, células inflamatorias y detritus celulares; esta situación se ve reducida en base líquida en la que se utiliza líquido fijador o preservante celular. Los más empleados son el ThinPrep y SurePath BD; en un estudio en el que se compararon estos fijadores para el procesamiento de especímenes con abundante moco, se evaluó la celularidad del componente escamoso que fue mayor en especímenes con máxima cantidad (2.000 UI/ul); se concluyó que especímenes procesados con SurePath $B D$ no mostraron disminución de la celularidad cuando se le adicionaba moco, en contraste con ThinPrep que sí reveló pérdida de la celularidad. ${ }^{5}$

En la citología en base líquida a diferencia de la convencional no se realiza extendido en la fase inicial, sino se transfiere el material (muestra) al líquido fijador o preservante, que incrementa la detección citológica de lesiones escamosas intraepiteliales y reducen el número de extendidos insatisfactorios. De acuerdo con el resultado de la revisión sistemática realizada en la base de estudios publicados de 1991 a 2007 a través de Pub Med/EmBase y metanálisis, la prueba de oro para la verificación es la colposcopia y la biopsia; en estos estudios se comparó la sensibilidad en base líquida para LIEA, LIEB y ASCUS, que corresponde a $57,1 \%, 79,1 \%$ y $90,4 \%$ con respecto a la convencional 
con $55,2 \%, 75,6 \%$ y $88,2 \%$. No hay diferencias estadísticas en sensibilidad y especificidad para neoplasias cervicales grado 2 entre la técnica convencional y la base líquida. ${ }^{6}$

Los citohistotecnólogos y patólogos prefieren la base líquida porque facilita la interpretación al observar células uniformes en monocapa; el tamizaje es más apropiado para aparatos automatizados, lo que la hace más costosa en términos de equipos y maquinaria para implementarla. ${ }^{6}$

Ambas técnicas ofrecen similar disponibilidad para detectar lesiones cervicales en base líquida. Después de la preparación para el diagnóstico citológico el remanente celular se usa para técnicas de biología molecular como la tipificación del VPH a través de microarreglos; el más usado es el SurePath $B D .^{7}$

\section{Métodos}

Para la recolección de datos en este estudio se llevó a cabo una campaña de promoción y prevención en Aldea Centro Sur en Bogotá D.C., el 2 de noviembre de 2010, con mujeres de vida sexual activa que firmaron consentimiento informado, mayores de 18 años. Se realizaron 29 citologías con muestra compartida (convencional/base líquida), siguiendo la técnica de su fabricante LucBase; los datos necesarios para el análisis fueron recolectados en el instrumento clasificado por el sistema Bethesda 2001.

Cumplidos estos requisitos se desarrolló el evento haciendo la toma de citología convencional utilizando para recolectar la muestra exocervical una espátula de Ayre y para la endocervical un citocepillo; una vez extendido el material biológico sobre el portaobjeto para la convencional, se guardó la espátula de Ayre y el citocepillo con el material residual en un frasco de polietileno con el líquido fijador (LucBase), se mezcló el contenido y se llevaron al laboratorio para la coloración de Papanicolaou. La lectura de ambas técnicas fue realizada por dos citohistólogas con varios años de experiencia y los resultados anormales por una patóloga.

\section{Resultados}

De las 29 muestras evaluadas por la técnica convencional, 16 presentaron material endocervical y/o de la zona de transformación (Tabla 1). De las 29 con técnica en base líquida solo una tuvo representación del canal endocervical y/o zona de transformación. Fueron insatisfactorias doce y rechazadas dos (Tabla 2). A todas las muestras de citología convencional se les realizó una lectura y se clasificaron tres casos como ASCUS. En base líquida se les pudo hacer interpretación a 14 muestras, doce fueron inadecuadas y dos rechazadas (Tabla 3). En lo referente al comparativo del estimativo de tiempo en la realización de cada una de las dos técnicas, se halló una diferencia de siete minutos en la citología en base líquida (Tabla 4).

\begin{tabular}{|l|c|c|}
\hline \multicolumn{2}{|c|}{$\begin{array}{c}\text { Tabla I. Calidad de la muestra y diagnóstico en } \\
\text { citogía convencional }\end{array}$} \\
\hline Calidad de la muestra & Negativo & Ascus \\
\hline $\begin{array}{l}\text { Satisfactoria con células endocervicales } \\
\text { y zona de transformación }\end{array}$ & 16 & 3 \\
$\begin{array}{l}\text { Satisfactoria sin células endocervicales } \\
\text { y zona de transformación }\end{array}$ & 10 & 0 \\
Insatisfactoria & 0 & 0 \\
Rechazada & 0 & 0 \\
\hline
\end{tabular}

\section{Tabla 2. Calidad de la muestra y diagnóstico} (base líquida)

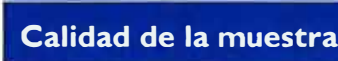

Sin lectura Negativo Ascus

Satisfactoria con células endocervicales y zona de transformación

Satisfactoria sin células endocervicales y zona de transformación

Insatisfactoria

Rechazada

\section{Tabla 3. Registro de lectura de citología convencional y en base líquida}

\begin{tabular}{|l|c|c|c|}
\hline Registro lectura & Negativas & Ascus & Total \\
\hline Citología convencional & 26 & 3 & 29 \\
Citología base líquida & 14 & 1 & 15 \\
\hline
\end{tabular}




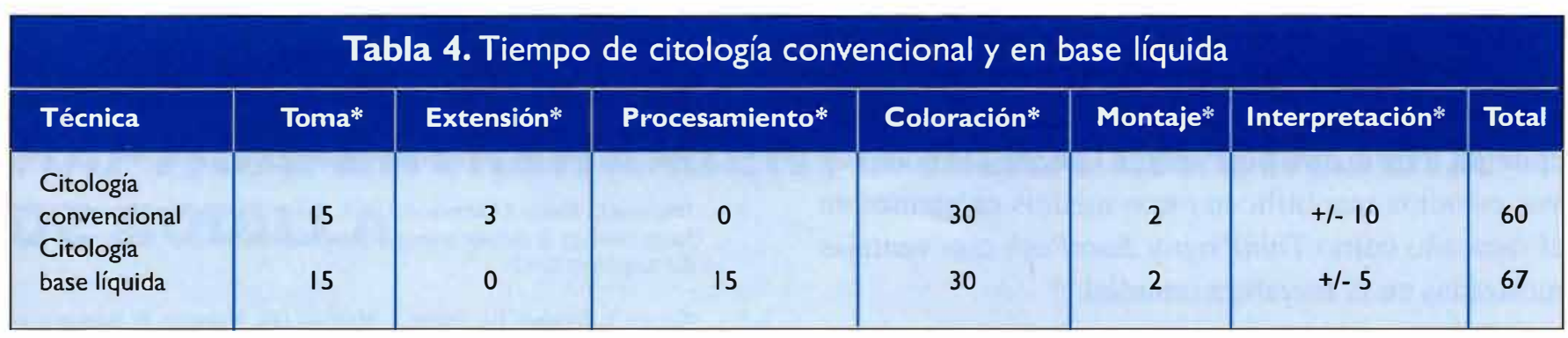

El tiempo se reporta en minutos y es aproximado.

\section{Discusión}

La experiencia de este estudio basado en extendidos citológicos con muestra compartida, realizando la citología convencional y después guardando la espátula de Ayre y el citocepillo en un vial que contenía $2 \mathrm{ml}$ de líquido preservante y transportador de la muestra (LucBase), para realizar la técnica en base líquida; este análisis permitió evaluar la calidad de la muestra en cuanto a elementos epiteliales presentes y hallazgos diagnósticos.

De las 29 muestras de tipo convencional 19/29 (65,5\%), presentaron células endocervicales y/o de la zona de transformación, en 10/29 (34,4\%), estaban ausentes y no se observaron extendidos insatisfactorios o rechazados. De las 29 en base líquida se encontraron 1/29 $(3,4 \%)$ con material endocervical y/o zona de transformación, 14/29 (48,2\%) satisfactorias sin células endocervicales y/o de la zona de transformación. 12/29 $(41,3 \%)$ fueron inadecuadas por escasa celularidad o por ser muy inflamatorias y no poderse analizar las células epiteliales y $2 / 29(6,8 \%)$ se rechazaron por acelulares. También se apreció lisis del material por la solución preservante que interfería en la lectura, pues los detritus hemáticos estaban en el fondo de la preparación.

La mayoría de los artículos científicos publicados concluyen que la técnica en base líquida tiene un porcentaje similar a la citología convencional, otros como "Split-Sample Analysis of Discarded Cells from Liquid-Based Pap Smear Sampling Devices"8 en el que la muestra fue compartida, concluyen que la calidad de la muestra disminuyó. Esta primera experiencia con base líquida en muestra compartida, revela que el mayor porcentaje de éxito con la convencional se explica por haberse realizado primero donde se encontraba la totalidad de las células recolectadas provenientes del cuello uterino, quedando una muestra poco representativa en la espátula de Ayre y en el citocepillo para el procesamiento por la técnica en base líquida.

En cuanto a la morfología no se halló diferencia entre las dos técnicas. La coloración de Papanicolaou fue buena en los dos procesos.

Respecto al hallazgo de atipia o detección de ASCUS, la convencional detectó tres casos mientras en base líquida fue uno. En la literatura revisada se evidencian dos nuevas tecnologías de apoyo diagnóstico disponibles en el mercado; ThinPrep y SurePath, aprobada por la Administración de Drogas y Alimentos de los Estados Unidos (FDA), que permiten el incremento en la detección de lesiones intraepiteliales escamosas y la reducción del número de extendidos convencionales insatisfactorios, recomendándose la verificación con colposcopia y biopsia si está indicado. Como desventaja para la citología en base líquida se anota la recolección inicial para el extendido convencional y el remanente para la base líquida. Por ello la transferencia de elementos diagnósticos es pobre, favoreciendo el método convencional.

Otra situación que favorece este hecho, es que el líquido fijador y preservante de LucBase no eliminó en su totalidad los elementos que alteran la visualización de las células, como son el moco y las células inflamatorias y hemáticas, puesto que la lisis de estos hizo parte del fondo de la preparación dando un aspecto sucio y 
es posible que haya incidido en una menor adhesión de las células epiteliales al portaobjeto y por lo tanto una de las causas del desprendimiento del material epitelial. Por lo anterior, se crea la necesidad de nuevos estudios que utilicen otros medios existentes en el mercado como ThinPrep y SurePath con ventajas mostradas en la literatura mundial. ${ }^{6,9}$

Dentro de las limitaciones del estudio encontramos el bajo número total de muestras evaluadas y la falta de estudios referentes a la implementación y uso de la citología en base líquida utilizando LucBase.

\section{Referencias}

1. Albujar P. Reseña histórica de la citología. Ginecol. Obstetr.[serie en Internet] 2001 abr [citado 30 Sep 2011 ] ; 47(2):[aprox. 3 p.]. Disponible en: http://sisbib. unms.edu.pe/bvrevistas/ginecologia/vol_47n2/resena_histo.htm.

2. Ricci P, Perucca E, Koljanin J, Baeriswyl E. Citología de base liquida: revisión de la historia y los estudios al respecto. Rev Chil obstét ginecol. [serie en In- ternet] 2004 [citado 30 Sep 2011]; 69 (3): ):[aprox. 7 p.]. Disponible en: http:// www.scielo.cl/pdf/rchog/v69n3/art 14.pdf

3. Wright PK. Comparison of SurePath and ThinPred liquid-based cervical cytology using positive predictive value, atypical predictive value and total predictive value as performance indicators. Cytopathology. 2010; 21:374-78.

4. Bergeron C, Bishop J, Lemarie A, Cas F, Ayivi J, Huynh B. Accuracy of ThinLayer cytology in patients undergoing cervical cone biopsy. Acta Cytol. 2001 Jul-Aug:45(4): 519-24

5. Kenyon S, Sweeney BJ, Happel J, Marchilli GE, Weinstein B, Schneider D Comparasion of BD Surepath and ThinPred pap systems in the processing of mucus rich specimens. Cancer Cytopathol. 2010 Oct 25;118(5):244-9.

6. Arbyn M, Bergeron C, Klinkhamer P, Martin-Hirsch P, Siebers AG, Bulten J. Liquid compared with conventional cervical cytology. Obstet Gynecol 2008; 111(1):167-77.

7. Taoka H, Yamamoto Y, Sakurai N, Fukuda M, Asakawa Y, Kurasaki A. Comparasion of convencional and liquid-based cytology, and human papillomavirus testing using SurePath preparation in Japan. Hum Cell. 2010 Nov;23(4):126-33

8. Ricci P. Perucca E. Koljnin J. Baeriswyl E Experiencia y manejo del purpura trombocitopenico idiopatico durante el embarazo. Rev. Chil. Obstet Ginecol. 2003;68(4):293-99.

9. Rinas AC, Mittman BW Jr, Le LV, Hartmann K, Cayless J, Singh HK. Split Sample Analysis of Discarded Cells from Liquid - Based Pap Smear Sampling Devices. Acta Cytol. 2006 Jan-Feb;50(1):55-62. 